ACCEPTED MANUSCRIPT

\title{
On the recursive solution of the quantum harmonic oscillator
}

To cite this article before publication: José M. Pérez-Jordá et al 2017 Eur. J. Phys. in press https://doi.org/10.1088/1361-6404/aa9584

\section{Manuscript version: Accepted Manuscript}

Accepted Manuscript is "the version of the article accepted for publication including all changes made as a result of the peer review process, and which may also include the addition to the article by IOP Publishing of a header, an article ID, a cover sheet and/or an 'Accepted Manuscript' watermark, but excluding any other editing, typesetting or other changes made by IOP Publishing and/or its licensors"

This Accepted Manuscript is @ 2017 European Physical Society.

During the embargo period (the 12 month period from the publication of the Version of Record of this article), the Accepted Manuscript is fully protected by copyright and cannot be reused or reposted elsewhere.

As the Version of Record of this article is going to be / has been published on a subscription basis, this Accepted Manuscript is available for reuse under a CC BY-NC-ND 3.0 licence after the 12 month embargo period.

After the embargo period, everyone is permitted to use copy and redistribute this article for non-commercial purposes only, provided that they adhere to all the terms of the licence https://creativecommons.org/licences/by-nc-nd/3.0

Although reasonable endeavours have been taken to obtain all necessary permissions from third parties to include their copyrighted content within this article, their full citation and copyright line may not be present in this Accepted Manuscript version. Before using any content from this article, please refer to the Version of Record on IOPscience once published for full citation and copyright details, as permissions will likely be required. All third party content is fully copyright protected, unless specifically stated otherwise in the figure caption in the Version of Record.

View the article online for updates and enhancements. 


\section{On the recursive solution of the quantumy} harmonic oscillator

José M. Pérez-Jordá*

Departament de Química Física, Universitat d'Alacant

$$
\text { E-03080, Alacant, Spain }
$$

October 11, 2017

This paper advocates writing the eigenfunctions of the harmonic oscillator without Hermite polynomials, by means of the following recurrence relation: $\Psi_{n}=\sqrt{2 \alpha / n} x \Psi_{n-1}-\sqrt{(n-1) / n} \Psi_{n-2}(n>0)$, with $\Psi_{0}=(\alpha / \pi)^{1 / 4} e^{-\alpha x^{2} / 2}$. It is easy to proof that these functions are eigenfunctions of the harmonic oscillator. It is shown that many of the exercises related to the harmonic oscillator found in textbooks can be solved in a simple and elegant way by using this recurrence relation. 


\section{Introduction}

The one-dimensional harmonic oscillator is an unavoidable model system in any quantum mechanics course, either introductory or advanced. There are several reasons for its popularity. First, it is an exactly solvable system. Second, students, once in possession of its solutions, can gain insight into the many concepts of the quantum world by practicing the myriad of exercises that pack textbooks[1-9] and journals[10, 11]. Third, despite its simplicity, the harmonic oscillator model is able to explain 'real-world' phenomena such as infrared spectroscopy or black-body radiation.

The eigenfunctions of a Hamiltonian $\hat{H}$ are the set of functions $\Psi_{n}$ that fulfill the time-independent Schrödinger's equation

$$
\hat{H} \Psi_{n}=E_{n} \Psi_{n},
$$

where $E_{n}$ is the eigenvalue corresponding to $\Psi_{n}$. For the one-dimensional harmonic oscillator, the Hamiltonian takes the form

$$
\hat{H}=\frac{1}{2 m} \hat{p}^{2}+\frac{1}{2} k \hat{x}^{2}
$$

where $\hat{x}$ and $\hat{p}$ are respectively the position and momentum operators,

$$
\begin{aligned}
& \hat{x}=x \\
& \hat{p}=-i \hbar \frac{d}{d x},
\end{aligned}
$$

$m$ is the particle's mass and $k$ the oscillator's force constant. It is known from the early days of quantum mechanics that the eigenvalues and eigenfunctions 
of the harmonic oscillator are given by

$$
\begin{aligned}
E_{n} & =h \nu\left(n+\frac{1}{2}\right), \quad n=0,1,2 \cdots \\
\Psi_{n}(x) & =\frac{1}{\sqrt{2^{n} n !}}\left(\frac{\alpha}{\pi}\right)^{1 / 4} H_{n}(x \sqrt{\alpha}) e^{-\alpha x^{2} / 2}
\end{aligned}
$$

where $\nu$ is the oscillator's frequency,

$$
\nu=\frac{1}{2 \pi} \sqrt{\frac{k}{m}},
$$

and

$$
\alpha=\frac{2 \pi \nu m}{\hbar}=\frac{\sqrt{k m}}{\hbar} .
$$

The symbol $H_{n}$ represents the Hermite polynomial of degree $n$.

Obtaining these eigenfunctions is not trivial. Two different approaches can be employed. One is the power-series method[1-3, 6-9], which requires lengthy manipulations of the equation, some of them controversial[12-14]. As an elegant alternative, but lengthy nonetheless, we can use the ladder or creation/annihilation operators $[2,4,5,7,8]$ method. In both cases, it is shown that the solutions fulfill a certain recurrence relation, out of which, and after some effort, the Hermite polynomials emerge.

One may wonder if the Hermite polynomials are really unavoidable. When solving this eigenproblem from scratch, one may consider stopping at the recurrence relation and circumvent the Hermite polynomials. Even if this proof from scratch is omitted, as may be the case in freshman courses, working with Hermite polynomials is tedious and error-prone, and the students have to be 
exposed to yet another concept, the properties of which are probably unknown to them and are often given without proof, increasing the feeling that quantum mechanics is some sort of revealed truth that cannot be understood, only believed.

This paper advocates to write the harmonic oscillator eigenfunctions by means of the recurrence relation

$$
\Psi_{n}=\sqrt{\frac{2 \alpha}{n}} x \Psi_{n-1}-\sqrt{\frac{n-1}{n}} \Psi_{n-2} \quad(n>0), \quad \Psi_{0}=\left(\frac{\alpha}{\pi}\right)^{1 / 4} e^{-\alpha x^{2} / 2} .
$$

This expression can be worked out from the recursion property of the Hermite polynomials[15], or from the properties of ladder operators[2]. We will prove in section 2 that the functions generated from Eq. (9) are indeed eigenfunctions of the harmonic oscillator, and then we will use this relation to solve some of the exercises usually found in textbooks. The manipulations needed to achieve these goals are simple enough to be suitable for non-advanced courses and do not require Hermite polynomials, nor solving differential equations, nor applying ladder operators. Some conclusions will be gathered in section 3 (in ànadvanced course, it may be advisable to prove that no function other than those generated from Eq. (9) can be an eigenfunction of the harmonic oscillator. This is done, to make the paper self-contained, in appendix C) 


\section{Properties and applications}

In this section we will study some properties of the harmonic oscillator eigenfunctions, and then use these properties to solve a selection of the exercises often found in textbooks. All this will be achieved entirely from Eq. (9), without requiring the use of Hermite polynomials nor any other advanced skill or concept.

\subsection{Position operator}

The result of acting on an eigenfunction $\Psi_{n}$ with the position operator can be obtained by means of Eqs. (3) and (9),

$$
\hat{x} \Psi_{n}=x \Psi_{n}=\sqrt{\frac{n}{2 \alpha}} \Psi_{n-1}+\sqrt{\frac{n+1}{2 \alpha}} \Psi_{n+1}, \quad n \geq 0 .
$$

Note that this equation, unlike the recursion relation in Eq. (9), is also valid for $n=0$.

\subsection{Momentum operator}

According to Eq: (4), to get the effect of $\hat{p}$ on any $\Psi_{n}$ obtained from Eq. (9), we need the first derivative of $\Psi_{n}$, which is given by

$$
\Psi_{n}^{\prime}=\frac{d \Psi_{n}}{d x}=\sqrt{\frac{\alpha n}{2}} \Psi_{n-1}-\sqrt{\frac{\alpha(n+1)}{2}} \Psi_{n+1}, \quad n \geq 0 .
$$

(see appendix A for the proof of this expression). Therefore, the effect of the momentum operator on $\Psi_{n}$ will be

$$
\hat{p} \Psi_{n}=-i \hbar \Psi_{n}^{\prime}=-i \hbar \sqrt{\frac{\alpha n}{2}} \Psi_{n-1}+i \hbar \sqrt{\frac{\alpha(n+1)}{2}} \Psi_{n+1}, \quad n \geq 0
$$




\subsection{Eigenfunctions of $\hat{H}$}

Let us check now that any function $\Psi_{n}$ from Eq. (9) is indeed an eigenfunction of $\hat{H}$ with eigenvalue $h \nu(n+1 / 2)$. We compute $\hat{x}^{2} \Psi_{n}$ by applying Eq. (10) twice,

$$
\begin{aligned}
\hat{x}^{2} \Psi_{n}= & \hat{x}\left(\hat{x} \Psi_{n}\right)=\hat{x}\left(\sqrt{\frac{n}{2 \alpha}} \Psi_{n-1}+\sqrt{\frac{n+1}{2 \alpha}} \Psi_{n+1}\right) \\
= & \sqrt{\frac{n}{2 \alpha}}\left(\sqrt{\frac{n-1}{2 \alpha}} \Psi_{n-2}+\sqrt{\frac{n}{2 \alpha}} \Psi_{n}\right)+ \\
& +\sqrt{\frac{n+1}{2 \alpha}}\left(\sqrt{\frac{n+1}{2 \alpha}} \Psi_{n}+\sqrt{\frac{n+2}{2 \alpha}} \Psi_{n+2}\right),
\end{aligned}
$$

which simplifies to

$$
\hat{x}^{2} \Psi_{n}=\frac{\sqrt{n(n-1)}}{2 \alpha} \Psi_{n-2}+\frac{2 n+1}{2 \alpha} \Psi_{n}+\frac{\sqrt{(n+1)(n+2)}}{2 \alpha} \Psi_{n+2} .
$$

Similarly, for $\hat{p}^{2} \Psi_{n}$, if we apply Eq. (12) twice we get

$$
\begin{aligned}
& \hat{p}^{2} \Psi_{n}=i \hbar \hat{p}\left(-\sqrt{\frac{\alpha n}{2}} \Psi_{n-1}+\sqrt{\frac{\alpha(n+1)}{2}} \Psi_{n+1}\right) \\
& =-\hbar^{2}\left(-\sqrt{\frac{\alpha n}{2}}\right)\left(-\sqrt{\frac{\alpha(n-1)}{2}} \Psi_{n-2}+\sqrt{\frac{\alpha n}{2}} \Psi_{n}\right)+ \\
& -\hbar^{2} \sqrt{\frac{\alpha(n+1)}{2}}\left(-\sqrt{\frac{\alpha(n+1)}{2}} \Psi_{n}+\sqrt{\frac{\alpha(n+2)}{2}} \Psi_{n+2}\right) \text {, }
\end{aligned}
$$

which gives

$$
\hat{p}^{2} \Psi_{n}=-\frac{\hbar^{2} \alpha}{2}\left(\sqrt{n(n-1)} \Psi_{n-2}-(2 n+1) \Psi_{n}+\sqrt{(n+1)(n+2)} \Psi_{n+2}\right)
$$


Now we use these results to apply $\hat{H}$ to $\Psi_{n}$. We get, according to Eq. (2)

$$
\begin{aligned}
\hat{H} \Psi_{n}= & \frac{1}{2 m} \hat{p}^{2} \Psi_{n}+\frac{1}{2} k \hat{x}^{2} \Psi_{n} \\
= & \left(-\frac{\hbar^{2} \alpha}{4 m}+\frac{k}{4 \alpha}\right) \sqrt{n(n-1)} \Psi_{n-2}+ \\
& +\left(\frac{\hbar^{2} \alpha}{4 m}+\frac{k}{4 \alpha}\right)(2 n+1) \Psi_{n}+ \\
& +\left(-\frac{\hbar^{2} \alpha}{4 m}+\frac{k}{4 \alpha}\right) \sqrt{(n+1)(n+2)} \Psi_{n+2}
\end{aligned}
$$

It turns out from the definition of $\alpha$ in Eq. (8), however, that

$$
\begin{aligned}
\frac{\hbar^{2} \alpha}{m} & =\frac{\hbar^{2}}{m} \frac{2 \pi \nu m}{\hbar}=h \nu \\
\frac{k}{\alpha} & =\frac{(\sqrt{k})^{2}}{\alpha}=\frac{\frac{\hbar^{2} \alpha^{2}}{m}}{\alpha}=\frac{\hbar^{2} \alpha}{m}=h \nu,
\end{aligned}
$$

so that we can simplify the expression of $\hat{H} \Psi_{n}$ to

$$
\hat{H} \Psi_{n}=h \nu\left(n+\frac{1}{2}\right) \Psi_{n},
$$

which proves that any $\Psi_{n}$ from Eq. (9) is an eigenfunction of $\hat{H}$ with the correct eigenvalue given by Eq. (5). Note, however, that the above discussion does not exclude the possibility that some functions other than those from Eq. (9) are eigenfunctions of $\hat{H}$ too. See appendix $\mathrm{C}$ for a rigorous proof.

\subsection{Parity}

A function $f$ is said to be even if $f(x)=f(-x)$, and odd if $f(x)=-f(-x)$. Let us consider the eigenfunctions defined by the recurrence relation in Eq. (9). It is clear that $\Psi_{0}$ is even. $\Psi_{1}$ is given by

$$
\Psi_{1}=\sqrt{2 \alpha} x \Psi_{0}
$$


and therefore is odd. It is easy to go on from here by inspecting Eq. (9): $\Psi_{2}$ is even, $\Psi_{3}$ odd, and so on. We conclude that an eigenfunction $\Psi_{n}$ is even if $n$ is even, but odd if $n$ is odd, which can be expressed by means of this formula

$$
\Psi_{n}(x)=(-1)^{n} \Psi_{n}(-x)
$$

\subsection{Orthonormality}

The set of eigenfunctions $\Psi_{n}$ form an orthonormal set,

Let us prove it by means of Eq. (9).

$$
\left\langle\Psi_{n} \mid \Psi_{m}\right\rangle=\delta_{n m}
$$

Two eigenfunctions $\Psi_{n}$ and $\Psi_{m}$ corresponding to different eigenvalues are orthogonal,

$$
\left\langle\Psi_{n} \mid \Psi_{m}\right\rangle=0, \quad n \neq m
$$

This is true not only for the harmonic oscillator, but for the eigenfunctions of any Hermitian operator. The proof is well known but we include it here for completeness. By the properties of Hermitian operators,

$$
\left\langle\Psi_{n}|\hat{H}| \Psi_{m}\right\rangle=\left\langle\Psi_{m}|\hat{H}| \Psi_{n}\right\rangle^{*}
$$

and, as $\Psi_{n}$ and $\Psi_{m}$ are eigenfunctions of $\hat{H}$ with respective (and different) real eigenvalues $E_{n}$ and $E_{m}$,

$$
E_{m}\left\langle\Psi_{n} \mid \Psi_{m}\right\rangle=E_{n}\left\langle\Psi_{m} \mid \Psi_{n}\right\rangle^{*}
$$


We know, in addition, that

$$
\left\langle\Psi_{m} \mid \Psi_{n}\right\rangle^{*}=\left\langle\Psi_{n} \mid \Psi_{m}\right\rangle
$$

so that

$$
E_{m}\left\langle\Psi_{n} \mid \Psi_{m}\right\rangle=E_{n}\left\langle\Psi_{n} \mid \Psi_{m}\right\rangle
$$

and therefore

$$
\left(E_{m}-E_{n}\right)\left\langle\Psi_{n} \mid \Psi_{m}\right\rangle=0
$$

As $E_{m}$ and $E_{n}$ are different, we must conclude that

$$
\left\langle\Psi_{n} \mid \Psi_{m}\right\rangle=0
$$

Let us prove now that any $\Psi_{n}$ is normalized. We use Eq. (9) to work on $\left\langle\Psi_{n} \mid \Psi_{n}\right\rangle$,

but, as $\Psi_{n}$ and $\Psi_{n-2}$ are orthogonal,

$$
\left\langle\Psi_{n} \mid \Psi_{n}\right\rangle=\sqrt{\frac{2 \alpha}{n}}\left\langle\Psi_{n} \mid x \Psi_{n-1}\right\rangle-\sqrt{\frac{n-1}{n}}\left\langle\Psi_{n} \mid \Psi_{n-2}\right\rangle
$$

$$
\left\langle\Psi_{n} \mid \Psi_{n}\right\rangle=\sqrt{\frac{2 \alpha}{n}}\left\langle\Psi_{n} \mid x \Psi_{n-1}\right\rangle=\sqrt{\frac{2 \alpha}{n}}\left\langle x \Psi_{n} \mid \Psi_{n-1}\right\rangle .
$$

Now we use Eq. (10),

$$
\left\langle\Psi_{n} \mid \Psi_{n}\right\rangle=\sqrt{\frac{2 \alpha}{n}}\left(\sqrt{\frac{n}{2 \alpha}}\left\langle\Psi_{n-1} \mid \Psi_{n-1}\right\rangle+\sqrt{\frac{n+1}{2 \alpha}}\left\langle\Psi_{n+1} \mid \Psi_{n-1}\right\rangle\right) .
$$

Again, as $\Psi_{n+1}$ and $\Psi_{n-1}$ are orthogonal,

$$
\left\langle\Psi_{n} \mid \Psi_{n}\right\rangle=\left\langle\Psi_{n-1} \mid \Psi_{n-1}\right\rangle
$$


so that, by repeated application of this equation, we arrive at

$$
\left\langle\Psi_{n} \mid \Psi_{n}\right\rangle=\left\langle\Psi_{0} \mid \Psi_{0}\right\rangle
$$

The ground-state eigenfunction is normalized, as proved in appendix B, and therefore

$$
\left\langle\Psi_{n} \mid \Psi_{n}\right\rangle=1
$$

which concludes the proof that the eigenfunctions of the harmonic oscillator given in Eq. (9) form an orthonormal set.

\subsection{Uncertainty relation}

Heisenberg's uncertainty relation for the position and momentum operators states that

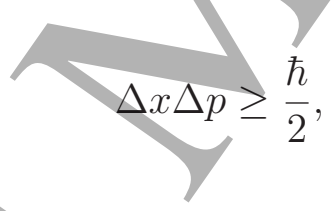

where

$$
\begin{aligned}
& \Delta x=\sqrt{\left\langle x^{2}\right\rangle-\langle x\rangle^{2}} \\
& \Delta p=\sqrt{\left\langle p^{2}\right\rangle-\langle p\rangle^{2}} .
\end{aligned}
$$

The expectations values $\langle x\rangle, \ldots,\left\langle p^{2}\right\rangle$ are computed from the normalized wave function $\Psi$ describing the system,

$$
\begin{aligned}
\langle x\rangle & =\langle\Psi|\hat{x}| \Psi\rangle=\langle\Psi \mid \hat{x} \Psi\rangle \\
\left\langle x^{2}\right\rangle & =\left\langle\Psi\left|\hat{x}^{2}\right| \Psi\right\rangle=\left\langle\Psi \mid \hat{x}^{2} \Psi\right\rangle \\
\langle p\rangle & =\langle\Psi|\hat{p}| \Psi\rangle=\langle\Psi \mid \hat{p} \Psi\rangle \\
\left\langle p^{2}\right\rangle & =\left\langle\Psi\left|\hat{p}^{2}\right| \Psi\right\rangle=\left\langle\Psi \mid \hat{p}^{2} \Psi\right\rangle .
\end{aligned}
$$


Next, we check the uncertainty relation for the eigenfunctions of the harmonic oscillator, $\Psi=\Psi_{n}$.

We begin by computing $\langle x\rangle$ by means of Eq. (10),

$$
\langle x\rangle=\sqrt{\frac{n}{2 \alpha}}\left\langle\Psi_{n} \mid \Psi_{n-1}\right\rangle+\sqrt{\frac{n+1}{2 \alpha}}\left\langle\Psi_{n}\right| \Psi_{n+1\rangle},
$$

and, recalling the orthonormality of $\Psi_{n}$, we get

$$
\langle x\rangle=0 .
$$

For $\left\langle x^{2}\right\rangle$, by using Eq. (14) and taking into account again the orthonormality of $\Psi_{n}$, we get

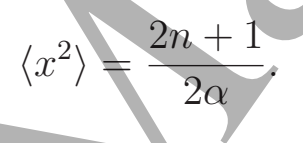

Similarly, by using Eqs. (12) and (16), we obtain

$$
\begin{aligned}
\langle p\rangle & =0 \\
\left\langle p^{2}\right\rangle & =\frac{\hbar^{2} \alpha(2 n+1)}{2} .
\end{aligned}
$$

We conclude that

$$
\Delta x \Delta p=\hbar\left(n+\frac{1}{2}\right) \geq \frac{\hbar}{2},
$$

which agrees with the uncertainty relation.

\subsection{Virial theorem}

The virjal theorem[9] states that

$$
\left\langle x \frac{d V}{d x}\right\rangle=2\langle T\rangle
$$


where $V$ represents the potential energy of the system and $T$ its kinetic energy. For the harmonic oscillator,

$$
\begin{aligned}
T & =\frac{1}{2 m} p^{2} \\
V & =\frac{1}{2} k x^{2},
\end{aligned}
$$

and as

$$
\left\langle x \frac{d V}{d x}\right\rangle=\langle x k x\rangle=2\langle V\rangle,
$$

the virial theorem takes the form

$$
\langle V\rangle=\langle T\rangle .
$$

The expectations values of $V$ and $T$ for any eigenfunction $\Psi_{n}$ are readily computed from Eqs. (46) and (48)

$$
\begin{aligned}
& \langle V\rangle=\frac{1}{2} k\left\langle x^{2}\right\rangle=\frac{k}{4 \alpha}(2 n+1) \\
& \langle T\rangle=\frac{1}{2 m}\left\langle p^{2}\right\rangle=\frac{\hbar^{2} \alpha}{4 m}(2 n+1),
\end{aligned}
$$

and taking into account Eqs. (18)-(19) we obtain

$$
\langle V\rangle=\langle T\rangle=\frac{h \nu}{2}\left(n+\frac{1}{2}\right)
$$

which agrees with the virial theorem.

\subsection{Selection rules}

The harmonic oscillator eigenfunctions $\Psi_{n}$ can be used to model the vibrational states of diatomic molecules[15, 16]. Assuming that the change of the 
molecule's dipole moment with internuclear distance is linear, a spectroscopic transition from state $\Psi_{n^{\prime \prime}}$ to state $\Psi_{n^{\prime}}$ is forbidden unless[16]

$$
\left\langle\Psi_{n^{\prime \prime}}|x| \Psi_{n^{\prime}}\right\rangle \neq 0
$$

This bracket is non-zero when the following selection rule is fulfilled[16],

$$
\Delta n=n^{\prime}-n^{\prime \prime}= \pm 1
$$

The proof is immediate from Eq. (10),

$$
\left\langle\Psi_{n^{\prime \prime}}|x| \Psi_{n^{\prime}}\right\rangle=\sqrt{\frac{n^{\prime}}{2 \alpha}}\left\langle\Psi_{n^{\prime \prime}} \mid \Psi_{n^{\prime}-1}\right\rangle+\sqrt{\frac{n^{\prime}+1}{2 \alpha}}\left\langle\Psi_{n^{\prime \prime}} \mid \Psi_{n^{\prime}+1}\right\rangle,
$$

which, by the orthogonality of the eigenvectors, is zero unless $n^{\prime \prime}=n^{\prime}-1$

$$
(\Delta n=+1) \text { or } n^{\prime \prime}=n^{\prime}+1(\Delta n=-1)
$$

\section{Conclusions}

The following conclusions can be drawn from this work.

- It is possible to write the eigenfunctions of the harmonic oscillator without having to use Hermite polynomials, by means of the recurrence relation shown in Eq. (9).

- It is easy (see subsection 2.3) to prove that these recursive functions are indeed eigenfunctions of the harmonic oscillator.

- It is simple and elegant, using the recursive formulation proposed in this work, to solve many of the typical exercises related to the harmonic oscillator often found in textbooks. 
- Eq. (9) can be proved from scratch by means of ladder operators (to make the paper self-contained, the proof is given in appendix $\mathrm{C}$ ).

\section{A Derivative of $\Psi_{n}$}

In order to prove Eq. (11) we will use the relation

$$
\alpha x \Psi_{n}+\Psi_{n}^{\prime}=\left(\alpha x+\frac{d}{d x}\right) \Psi_{n}=\sqrt{2 \alpha n} \Psi_{n-1}, \quad n \geq 0,
$$

which is proved next.

For $n=0$, we operate on the expression of $\Psi_{0}$ given in Eq. (9),

$$
\alpha x \Psi_{0}+\Psi_{0}^{\prime}=\left(\alpha x+\frac{d}{d x}\right)\left[\left(\frac{\alpha}{\pi}\right)^{1 / 4} e^{-\alpha x^{2} / 2}\right]=\alpha x \Psi_{0}-\alpha x \Psi_{0}=0,
$$

which agrees with Eq. (61)

For $n>0$, we operate on the expression of $\Psi_{n}$ from Eq. (9),

$$
\begin{aligned}
\alpha x \Psi_{n}+\Psi_{n}^{\prime}= & \left(\alpha x+\frac{d}{d x}\right)\left(\sqrt{\frac{2 \alpha}{n}} x \Psi_{n-1}-\sqrt{\frac{n-1}{n}} \Psi_{n-2}\right) \\
= & \alpha x\left(\sqrt{\frac{2 \alpha}{n}} x \Psi_{n-1}-\sqrt{\frac{n-1}{n}} \Psi_{n-2}\right)+ \\
& +\sqrt{\frac{2 \alpha}{n}} \Psi_{n-1}+\sqrt{\frac{2 \alpha}{n}} x \Psi_{n-1}^{\prime}-\sqrt{\frac{n-1}{n}} \Psi_{n-2}^{\prime} \\
= & \left.\sqrt{\frac{2 \alpha}{n} x(\alpha x} \Psi_{n-1}+\Psi_{n-1}^{\prime}\right)- \\
& -\sqrt{\frac{n-1}{n}}\left(\alpha x \Psi_{n-2}+\Psi_{n-2}^{\prime}\right)+\sqrt{\frac{2 \alpha}{n}} \Psi_{n-1} .
\end{aligned}
$$

The terms in parenthesis are the $n-1$ and $n-2$ counterparts of $\alpha x \Psi_{n}+\Psi_{n}^{\prime}$.

This suggests the use of mathematical induction: we assume that Eq. (61) 
have been proved for $n-1$ and $n-2$, and then replace them in Eq. (63) (note that for $n=1$, only $\alpha x \Psi_{0}+\Psi_{0}^{\prime}$ is needed),

$$
\begin{aligned}
\alpha x \Psi_{n}+\Psi_{n}^{\prime}= & \sqrt{\frac{4 \alpha^{2}(n-1)}{n}} x \Psi_{n-2}- \\
& -\sqrt{\frac{2 \alpha(n-1)(n-2)}{n}} \Psi_{n-3}+\sqrt{\frac{2 \alpha}{n} \Psi_{n-1}}
\end{aligned}
$$

Next we replace the value of $x \Psi_{n-2}$, which can obtained from Eq. (10),

$$
\begin{aligned}
\alpha x \Psi_{n}+\Psi_{n}^{\prime}= & \sqrt{\frac{4 \alpha^{2}(n-1)}{n}}\left(\sqrt{\frac{n-2}{2 \alpha}} \Psi_{n-3}+\sqrt{\frac{n-1}{2 \alpha}} \Psi_{n-1}\right)- \\
& -\sqrt{\frac{2 \alpha(n-1)(n-2)}{n} \Psi_{n-3}+\sqrt{\frac{2 \alpha}{n}} \Psi_{n-1}} \\
= & (n-1) \sqrt{\frac{2 \alpha}{n}} \Psi_{n-1}+\sqrt{\frac{2 \alpha}{n}} \Psi_{n-1}=\sqrt{2 \alpha n} \Psi_{n-1},
\end{aligned}
$$

which agrees with Eq. (61). We conclude, by the logic of mathematical induction, that Eq. (61) is true for any $n \geq 0$ : From the case $n=0$ we can prove the case $n=1$, from $n=0$ and $n=1$ we can prove the case $n=2$, and so on.

We use Eq. (61) to get the derivative of $\Psi_{n}$,

$$
\Psi_{n}^{\prime}=\sqrt{2 \alpha n} \Psi_{n-1}-\alpha x \Psi_{n}
$$

and, after replacing the value of $x \Psi_{n}$ from Eq. (10), we obtain Eq. (11),

$$
\begin{aligned}
& =\sqrt{2 \alpha n} \Psi_{n-1}-\alpha\left(\sqrt{\frac{n}{2 \alpha}} \Psi_{n-1}+\sqrt{\frac{n+1}{2 \alpha}} \Psi_{n+1}\right) \\
& =\sqrt{\frac{\alpha n}{2}} \Psi_{n-1}-\sqrt{\frac{\alpha(n+1)}{2}} \Psi_{n+1} .
\end{aligned}
$$




\section{B Normalization of $\Psi_{0}$}

We will check here that the ground-state eigenfunction $\Psi_{0}$ given in Eq. (9) is normalized. Let us compute the bracket $\left\langle\Psi_{0} \mid \Psi_{0}\right\rangle$,

$$
\left\langle\Psi_{0} \mid \Psi_{0}\right\rangle=\sqrt{\frac{\alpha}{\pi}} \int_{-\infty}^{\infty} e^{-\alpha x^{2}} d x
$$

The integral above can be readily found in many tables of integrals [17], but, for the sake of completeness, we will compute it here. We express it in terms of a two-dimensional integral,

$$
\begin{aligned}
\int_{-\infty}^{\infty} e^{-\alpha x^{2}} d x & =\sqrt{\left(\int_{-\infty}^{\infty} e^{-\alpha x^{2}} d x\right)\left(\int_{-\infty}^{\infty} e^{-\alpha y^{2}} d y\right)} \\
& =\sqrt{\left(\int_{-\infty}^{\infty} \int_{-\infty}^{\infty} e^{\alpha\left(x^{2}+y^{2}\right)} d x d y\right)}
\end{aligned}
$$

which we can simplify, first by using polar coordinates,

$$
\int_{-\infty}^{\infty} e^{-\alpha x^{2}} d x=\sqrt{\int_{0}^{\infty} \int_{0}^{2 \pi} e^{-\alpha r^{2}} r d r d \varphi}=\sqrt{2 \pi \int_{0}^{\infty} e^{-\alpha r^{2}} r d r}
$$

then by the change of variable $t=\alpha r^{2}$,

$$
\int_{-\infty}^{\infty} e^{-\alpha x^{2}} d x=\sqrt{\frac{\pi}{\alpha} \int_{0}^{\infty} e^{-t} d t}=\sqrt{\frac{\pi}{\alpha}\left[-e^{-t}\right]_{0}^{\infty}}=\sqrt{\frac{\pi}{\alpha}}
$$

We conclude that

$$
\left\langle\Psi_{0} \mid \Psi_{0}\right\rangle=1
$$

\section{Eq. (9) gives all possible eigenfunctions}

In this section we will prove that any function given by Eq. (9) is a normalized eigenfunction of the harmonic oscillator, and, conversely, that any normalized 
eigenfunction can be obtained from Eq. (9). We will use the ladder operators method[2].

\section{C.1 $\hat{a}$ and $\hat{a}^{\dagger}$ operators}

The ladder or creation/annihilation operators can be defined as

$$
\begin{aligned}
\hat{a} & =\frac{\alpha \hbar}{\sqrt{2 m}} \hat{x}+\frac{i}{\sqrt{2 m}} \hat{p} \\
\hat{a}^{\dagger} & =\frac{\alpha \hbar}{\sqrt{2 m}} \hat{x}-\frac{i}{\sqrt{2 m}} \hat{p}
\end{aligned}
$$

(note that $\hat{a}$ and $\hat{a}^{\dagger}$ are usually defined to be dimensionless operators, and differ from the above expressions by a constant). Operators $\hat{a}$ and $\hat{a}^{\dagger}$ are each other adjoints.

\section{C.2 $\hat{a} \hat{a}^{\dagger}$ and $\hat{a}^{\dagger} \hat{a}$ operators}

Let us apply $\hat{a} \hat{a}^{\dagger}$ to an arbitrary wave function $\Psi$ (no necessarily an eigenfunction of $\hat{H})$ :

$$
\begin{aligned}
\hat{a} \hat{a}^{\dagger} \Psi & =\left(\frac{\alpha \hbar}{\sqrt{2 m}} \hat{x}+\frac{i}{\sqrt{2 m}} \hat{p}\right)\left(\frac{\alpha \hbar}{\sqrt{2 m}} \hat{x}-\frac{i}{\sqrt{2 m}} \hat{p}\right) \Psi \\
& =\frac{\alpha^{2} \hbar^{2}}{2 m} \hat{x}^{2} \Psi-i \frac{\alpha \hbar}{2 m} \hat{x} \hat{p} \Psi+i \frac{\alpha \hbar}{2 m} \hat{p} \hat{x} \Psi+\frac{1}{2 m} \hat{p}^{2} \Psi \\
& =\frac{\alpha^{2} \hbar^{2}}{2 m} \hat{x}^{2} \Psi+\frac{1}{2 m} \hat{p}^{2} \Psi-i \frac{\alpha \hbar}{2 m}[\hat{x}, \hat{p}] \Psi,
\end{aligned}
$$

which, from the definition of $\alpha$ in Eq. (8) and the expression of $\hat{H}$ in Eq. (2) becomes

$$
\hat{a} \hat{a}^{\dagger} \Psi=\frac{1}{2} k \hat{x}^{2} \Psi+\frac{1}{2 m} \hat{p}^{2} \Psi-i \frac{\alpha \hbar}{2 m}[\hat{x}, \hat{p}] \Psi=\hat{H} \Psi-i \frac{\alpha \hbar}{2 m}[\hat{x}, \hat{p}] \Psi .
$$


We can compute the commutator $[\hat{x}, \hat{p}]$ from the definition of the operators $\hat{x}$ and $\hat{p}$ in Eqs. (3)-(4),

$$
[\hat{x}, \hat{p}] \Psi=\hat{x} \hat{p} \Psi-\hat{p} \hat{x} \Psi=-i \hbar x \frac{d \Psi}{d x}+i \hbar \frac{d(x \Psi)}{d x}=i \hbar \Psi
$$

so that

$$
\hat{a} \hat{a}^{\dagger} \Psi=\hat{H} \Psi+\frac{\alpha \hbar^{2}}{2 m} \Psi
$$

and, from Eq. (18),

$$
\hat{a} \hat{a}^{\dagger} \Psi=\hat{H} \Psi+\frac{h \nu}{2} \Psi
$$

As $\Psi$ is an arbitrary function, we conclude that

$$
\hat{a} \hat{a}^{\dagger}=\hat{H}+\frac{h \nu}{2}
$$

Similarly, we can prove that

$$
\hat{a}^{\dagger} \hat{a}=\hat{H}-\frac{h \nu}{2}
$$

\section{C.3 Properties of $\hat{a} \Psi, \hat{H} \Psi=E_{\Psi} \Psi$}

Let us assume that $\Psi$ is an eigenfunction of $\hat{H}$ with eigenvalue $E_{\Psi}$,

$$
\hat{H} \Psi=E_{\Psi} \Psi
$$

Let us study how the function $\hat{a} \Psi$ is transformed by operator $\hat{H}$ : we have, from Eqs. (81) and (82), that

$$
\begin{aligned}
\hat{H}(\hat{a} \Psi) & =\left(\hat{a} \hat{a}^{\dagger}-\frac{h \nu}{2}\right)(\hat{a} \Psi)=\hat{a}\left(\hat{a}^{\dagger} \hat{a}\right) \Psi-\frac{h \nu}{2} \hat{a} \Psi \\
& =\hat{a}\left(\hat{H}-\frac{h \nu}{2}\right) \Psi-\frac{h \nu}{2} \hat{a} \Psi=\hat{a} \hat{H} \Psi-h \nu(\hat{a} \Psi)
\end{aligned}
$$


so that, from Eq. (83), we get

$$
\hat{H}(\hat{a} \Psi)=\left(E_{\Psi}-h \nu\right)(\hat{a} \Psi) .
$$

This means that $\hat{a} \Psi$ is either zero or an eigenfunction of $\hat{H}$ with eigenvalue $E_{\Psi}-h \nu$

Let us denote the ground-state eigenfunction by $\Psi_{0}$ and its eigenvalue by $E_{\Psi_{0}}$. The function $\hat{a} \Psi_{0}$ cannot be an eigenfunction of $\hat{H}$ because, according to Eq. (85), it would have $E_{\Psi_{0}}-h \nu$ as eigenvalue, which is below the ground-state energy. Therefore,

$$
\hat{a} \Psi_{0}=0 \text {. }
$$

It is easy to obtain, from this equation and Eq. (82), the ground-state eigenvalue:

$$
\hat{H} \Psi_{0}=\left(\hat{a}^{\dagger} \widehat{a}+\frac{h \nu}{2}\right) \Psi_{0}=\hat{a}^{\dagger}\left(\hat{a} \Psi_{0}\right)+\frac{h \nu}{2} \Psi_{0}=\frac{h \nu}{2} \Psi_{0} .
$$

We conclude that $E_{\Psi_{0}}=h \nu / 2$, which agrees with $E_{0}$ in Eq. (5).

Next, we will prove that for any excited state $\Psi, \hat{a} \Psi$ cannot be zero. We proceed by contradiction, and assume that for some excited $\Psi, \hat{a} \Psi=0$. Then, by/repeating the manipulations carried out in Eq. (87), we find out that the eigenvalue of $\Psi$ should be equal to the eigenvalue $E_{0}$ of the groundstate, which is in contradiction with $\Psi$ being an excited state. We conclude, according to Eqs. (85) and (86), that $\hat{a} \Psi$ is zero when $\Psi$ is the ground state, but it is and eigenfunction of $\hat{H}$ with eigenvalue $E_{\Psi}-h \nu$ when $\Psi$ is an excited 
state,

$$
\hat{H} \Psi=E_{\Psi} \Psi \quad \longrightarrow \quad \begin{cases}\text { if } \Psi=\Psi_{0}: & \hat{a} \Psi=0 \\ \text { if } \Psi \neq \Psi_{0}: & \hat{a} \Psi \neq 0,\end{cases}
$$

\section{C.4 Properties of $\hat{a}^{\dagger} \Psi, \hat{H} \Psi=E_{\Psi} \Psi$}

Given any eigenfunction $\Psi$ of $\hat{H}$, let us study now how $\hat{a}^{\dagger} \Psi$ is transformed by $\hat{H}$ : we have, from Eqs. (81) and (82), that

$$
\begin{aligned}
\hat{H}\left(\hat{a}^{\dagger} \Psi\right) & =\left(\hat{a}^{\dagger} \hat{a}+\frac{h \nu}{2}\right)\left(\hat{a}^{\dagger} \Psi\right)=\hat{a}^{\dagger}\left(\hat{a} \hat{a}^{\dagger}\right) \Psi+\frac{h \nu}{2} \hat{a}^{\dagger} \Psi \\
& =\hat{a}^{\dagger}\left(\hat{H}+\frac{h \nu}{2}\right) \Psi+\frac{h \nu}{2} \hat{a}^{\dagger} \Psi=\hat{a}^{\dagger} \hat{H} \Psi+h \nu\left(\hat{a}^{\dagger} \Psi\right),
\end{aligned}
$$

so that, from Eq. (83), we get

$$
\hat{H}\left(\hat{a}^{\dagger} \Psi\right)=\left(E_{\Psi}+h \nu\right)\left(\hat{a}^{\dagger} \Psi\right)
$$

This means that $\hat{a}^{\dagger} \Psi$ is either zero or an eigenfunction of $\hat{H}$ with eigenvalue $E_{\Psi}+h \nu$. We will prove next that $\hat{a}^{\dagger} \Psi$ cannot be zero, and therefore it has to be an eigenfunction of $\hat{H}$.

We will proceed by contradiction, and assume that, for some eigenfunction $\Psi, \hat{a}^{\dagger} \Psi$ is zero,

Then, from Eq. (81)

$$
\hat{a}^{\dagger} \Psi=0
$$

$$
\hat{H} \Psi=\left(\hat{a} \hat{a}^{\dagger}-\frac{\hbar \nu}{2}\right) \Psi=\hat{a}\left(\hat{a}^{\dagger} \Psi\right)-\frac{\hbar \nu}{2} \Psi=-\frac{\hbar \nu}{2} \Psi
$$


that is, $\Psi$ would be an eigenfunction of $\hat{H}$ with eigenvalue $-h \nu / 2$, but this is impossible because it would be below the ground-state eigenvalue obtained in Eq. (87). We conclude that $\hat{a}^{\dagger} \Psi$ is an eigenfunction of $\hat{H}$ with eigenvalue $E_{\Psi}+h \nu$

$$
\hat{H} \Psi=E_{\Psi} \Psi \quad \longrightarrow \quad \hat{a}^{\dagger} \Psi \neq 0, \quad \hat{H}\left(\hat{a}^{\dagger} \Psi\right)=\left(E_{\Psi}+h \nu\right)\left(\hat{a}^{\dagger} \Psi\right) .
$$

\section{C.5 Ground-state eigenfunction}

To obtain the ground-state eigenfunction, we have to solve Eq. (86). From the definition of $\hat{a}$ in Eq. (74) we get

$$
i \hat{p} \Psi_{0}=-\alpha \hbar \hat{x} \Psi_{0}
$$

so that, taking into account the expression of $\hat{x}$ and $\hat{p}$ in Eqs. (3)-(4),

$$
\frac{d \Psi_{0}}{d x}=-\alpha x \Psi_{0} .
$$

Solving this ordinary differential equation is simple,

which we integrate to

$$
\frac{d \Psi_{0}}{\Psi_{0}}=-\alpha x d x
$$

$$
\int_{C}^{\Psi_{0}} \frac{d \Psi_{0}}{\Psi_{0}}=-\alpha \int_{0}^{x} x d x
$$

where $C$ is an integration constant. After computing the integrals we get

$$
\ln \Psi_{0}-\ln C=-\frac{\alpha x^{2}}{2}
$$


so that

$$
\Psi_{0}=C e^{-\alpha x^{2} / 2}
$$

We can fix the value of $C$ by normalization. The normalized expression of $\Psi_{0}$ is (see appendix B)

$$
\Psi_{0}=\left(\frac{\alpha}{\pi}\right)^{1 / 4} e^{-\alpha x^{2} / 2}
$$

which agrees with the expression given in Eq. (9),

It is important to note that $\Psi_{0}$ is, up to a mormalization constant, the only possible solution of Eq. (86). This means that the ground state is nondegenerate.

\section{C.6 Excited-state eigenvalues}

We have seen, in Eq. (87), that the ground-state eigenvalue is $E_{0}=h \nu / 2$, and we have proved in subsection C.5 that it is non-degenerate. Let us extend this study to excited-state eigenvalues.

Let us begin with the ground-state eigenfunction $\Psi_{0}$, which fulfills

$$
\hat{H} \Psi_{0}=\frac{h \nu}{2} \Psi_{0}
$$

Then, according to Eq. (93), $\hat{a}^{\dagger} \Psi_{0}$ will be an excited eigenfunction of $\hat{H}$ with eigenvalue $3 h v / 2$

$$
\hat{H}\left(\hat{a}^{\dagger} \Psi_{0}\right)=\frac{3 h \nu}{2}\left(\hat{a}^{\dagger} \Psi_{0}\right)
$$

and, by $n$ successive applications of operator $\hat{a}^{\dagger}$ over $\Psi_{0}$, we will arrive at

$$
\hat{H} \Psi_{n}=E_{n} \Psi_{n}, \quad E_{n}=h \nu\left(n+\frac{1}{2}\right), \quad \Psi_{n}=\left(\hat{a}^{\dagger}\right)^{n} \Psi_{0}, \quad n=0,1, \cdots
$$


that is, $\Psi_{n}=\left(\hat{a}^{\dagger}\right)^{n} \Psi_{0}$ is an eigenfunction of $\hat{H}$ with eigenvalue $h \nu(n+1 / 2)$. This agrees with the well-known eigenvalues of the harmonic oscillator given in Eq. (5).

Having proved that $h \nu(n+1 / 2)$, with $n$ a non-negative integer, is an eigenvalue of $\hat{H}$, now we have to prove that no value other than $h \nu(n+1 / 2)$ is an eigenvalue. Negative integer values of $n$ are out of the question, because they will yield eigenvalues below the ground state, which is absurd. Let us try now non-integer values of $n$, and assume that there is an excited-state eigenfunction $\Psi$ with an eigenvalue $E_{\Psi}$ located between $E_{n}$ and $E_{n+1}$,

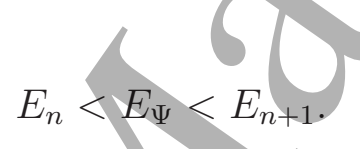

Now we apply the $\hat{a}$ operator to $\Psi n$ times, to get, according to Eq. (88), an eigenfunction $\hat{a}^{n} \Psi$ with eigenvalue

which is still above the ground state,

$$
E_{0}<E_{\hat{a}^{n} \Psi}<E_{1}
$$

As $E_{\hat{a}^{n} \Psi}$ is above the ground state, the eigenfunction $\hat{a}^{n} \Psi$ is an excited state, and, according to Eq. (88), if we apply $\hat{a}$ one more time, we will get an eigenfunction $\hat{a}^{n+1} \Psi$ with an eigenvalue below the ground-state, which is impossible. Therefore, the only possible eigenvalues of the harmonic oscillator are those given by Eqs. (5). 
We have seen in subsection C.5 that the ground-state is non-degenerate. We will prove next that excited states are also non-degenerate. We will proceed by contradiction, and assume that the first excited state is degenerate, with two eigenfunctions $\Psi_{1}$ and $\bar{\Psi}_{1}$ with the same eigenvalue $E_{1}=3 h \nu / 2$. $\Psi_{1}$ and $\bar{\Psi}_{1}$ must be linearly independent,

$$
\bar{\Psi}_{1} \neq k \Psi_{1}
$$

for any constant $k$. Next, we apply the $\hat{a}$ operator to both $\Psi_{1}$ and $\bar{\Psi}_{1}$, and obtain, according to Eq. (88), the respective eigenfunctions $\hat{a} \Psi_{1}$ and $\hat{a} \bar{\Psi}_{1}$, both with eigenvalue $E_{0}=h \nu / 2$, which/correspond to the ground state. However, as the ground state is non-degenerate, $\hat{a} \Psi_{1}$ and $\hat{a} \bar{\Psi}_{1}$ must be linearly dependent, that is,

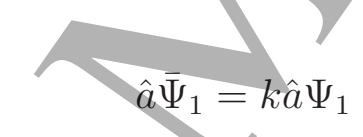

for some constant $k$. Now we apply the $\hat{a}^{\dagger}$ operator to both sides of this relation,

use Eq. (82),

$$
\hat{a}^{\dagger} \hat{a} \bar{\Psi}_{1}=k \hat{a}^{\dagger} \hat{a} \Psi_{1}
$$

$$
\begin{aligned}
\left(\frac{3 h \nu}{2}-\frac{h \nu}{2}\right) \bar{\Psi}_{1} & =k\left(\frac{3 h \nu}{2}-\frac{h \nu}{2}\right) \Psi_{1} \\
\bar{\Psi}_{1} & =k \Psi_{1}
\end{aligned}
$$


which is in contradiction with Eq. (107). Therefore, the first excited state $\Psi_{1}$ must be non-degenerate. We can now repeat the proof for $\Psi_{2}$, then for $\Psi_{3}$, and so on. We conclude that all the states of the harmonic oscillator are non-degenerate.

\section{C.7 Normalization}

Given an eigenfunction $\Psi_{n}$, we know, from Eqs (88) and (93), that $\hat{a} \Psi_{n}$ and $\hat{a}^{\dagger} \Psi_{n}$ are the eigenfunctions corresponding to the eigenvalues $E_{n-1}$ and $E_{n+1}$, and therefore should be respectively proportional to $\Psi_{n-1}$ and $\Psi_{n+1}$,

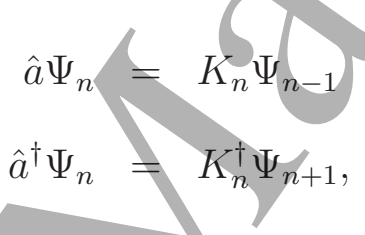

where $K_{n}$ and $K_{n}^{\dagger}$ are constants (note that $K_{n}^{\dagger}$ is not the 'adjoint' of $K_{n}$, but the constant corresponding to $\left.\hat{a}^{\dagger}\right)$. We can find out the values of these constants by assuming that all three $\Psi_{n}, \Psi_{n-1}$, and $\Psi_{n+1}$ are normalized.

Let us compute $\left\langle\hat{a} \Psi_{n} \mid \hat{a} \Psi_{n}\right\rangle$ with the help of Eqs. (82) and (5),

$$
\left\langle\hat{a} \Psi_{n} \mid \hat{a} \Psi_{n}\right\rangle=\left\langle\Psi_{n}\left|\hat{a}^{\dagger} \hat{a}\right| \Psi_{n}\right\rangle=\left\langle\Psi_{n}\left|\hat{H}-\frac{h \nu}{2}\right| \Psi_{n}\right\rangle=h \nu n,
$$

where we have used the fact that $\Psi_{n}$ is normalized. On the other hand, we can also compute this bracket by means of Eq. (113),

so that, by equating the two previous equations, we get

$$
K_{n}=\sqrt{h \nu n} .
$$


We proceed similarly for $K_{n}^{\dagger}$, but using Eqs. (81) and (5),

$$
\begin{aligned}
\left\langle\hat{a}^{\dagger} \Psi_{n} \mid \hat{a}^{\dagger} \Psi_{n}\right\rangle & =\left\langle\Psi_{n}\left|\left(\hat{a}^{\dagger}\right)^{\dagger} \hat{a}^{\dagger}\right| \Psi_{n}\right\rangle=\left\langle\Psi_{n}\left|\hat{a} \hat{a}^{\dagger}\right| \Psi_{n}\right\rangle \\
& =\left\langle\Psi_{n}\left|\hat{H}+\frac{h \nu}{2}\right| \Psi_{n}\right\rangle=h \nu(n+1)
\end{aligned}
$$

and, from Eq. (114),

$$
\left\langle\hat{a}^{\dagger} \Psi_{n} \mid \hat{a}^{\dagger} \Psi_{n}\right\rangle=\left(K_{n}^{\dagger}\right)^{2}
$$

which gives us

$$
K_{n}^{\dagger}=\sqrt{h \nu(n+1)}
$$

Summing up,

$$
\begin{aligned}
\hat{a} \Psi_{n} & =\sqrt{h \nu n} \Psi_{n-1} \\
\hat{a}^{\dagger} \Psi_{n} & =\sqrt{h \nu(n+1)} \Psi_{n+1} .
\end{aligned}
$$

Note that for the ground state $\Psi_{0}$, and according to Eq. (88), $\hat{a} \Psi_{0}$ should be zero, which agrees with Eq. (121).

\section{C.8 Proof of the recurrence relation}

To complete the derivation of the harmonic oscillator solutions, we have to prove the recurrence relation for $\Psi_{n}$ given in Eq. (9). First we express the position operator $\hat{x}$ in terms of the operators $\hat{a}$ and $\hat{a}^{\dagger}$, which is easily done by taking into account their definition in Eqs. (74)-(75),

$$
\hat{x}=\frac{\sqrt{m}}{\alpha \hbar \sqrt{2}}\left(\hat{a}+\hat{a}^{\dagger}\right),
$$


and then, by means of Eqs. (121)-(122), we apply it to $\Psi_{n-1}$,

$$
\hat{x} \Psi_{n-1}=\frac{\sqrt{m}}{\alpha \hbar \sqrt{2}}\left(\sqrt{h \nu(n-1)} \Psi_{n-2}+\sqrt{h \nu n} \Psi_{n}\right), \quad n>0
$$

Next, from the definition of $\alpha$ in Eq. (8),

$$
\frac{\sqrt{m}}{\alpha \hbar \sqrt{2}} \sqrt{h \nu}=\frac{\sqrt{2 \pi \nu m \hbar}}{\alpha \hbar \sqrt{2}}=\frac{\sqrt{\frac{2 \pi \nu m}{\hbar}}}{\alpha \sqrt{2}}=\frac{\sqrt{\alpha}}{\alpha \sqrt{2}}=\frac{1}{\sqrt{2 \alpha}}
$$

so that

$$
\hat{x} \Psi_{n-1}=\frac{1}{\sqrt{2 \alpha}}\left(\sqrt{n-1} \Psi_{n-2}+\sqrt{n} \Psi_{n}\right), \quad n>0 .
$$

Noting that, according to Eq. (3), $\hat{x}=x$, we finally get

$$
\Psi_{n}=\sqrt{\frac{2 \alpha}{n}} x \Psi_{n-1}-\sqrt{\frac{n-1}{n}} \Psi_{n-2}, \quad n>0,
$$

which proves Eq. (9).

\section{References}

[1] Leonard I. Schiff. Quantum Mechanics. McGraw-Hill, Singapore, third edition, 1968.

[2] Claude Cohen-Tannoudji, Bernard Diu, and Franck Laloë. Quantum Mechanics, volume 1. Wiley, New York, 1977.

[3] Linus Pauling and E. Bright Wilson, Jr. Introduction to Quantum Mechanics with Applications to Chemistry. Dover, New York, 1985.

[4] David Bohm. Quantum Theory. Dover, New York, 1989. 
[5] Frank L. Pilar. Elementary Quantum Chemistry. Dover, Mineola, second edition, 1990.

[6] John P. Lowe. Quantum Chemistry. Academic Press, San Diego, second edition, 1993.

[7] R. Shankar. Principles of Quantum Mechanics. Kluwer Academic, New York, second edition, 1994.

[8] Nouredine Zettili. Quantum Mechanics. Wiley, second edition, 2009.

[9] Ira N. Levine. Quantum Chemistry. Pearson, Boston, seventh edition, 2014.

[10] Pedro H. F. Nogueira and Antonio S. de Castro. Revisiting the quantum harmonic oscillator via unilateral Fourier transforms. Eur. J. Phys., 37:015402, 2016 .

[11] Riccardo Borghi. Quantum harmonic oscillator: an elementary derivation of the energy spectrum. Eur. J. Phys., 38:025404, 2017.

[12] H. A. Buchdahl. Remark on the solutions of the harmonic oscillator equation. Am. J. Phys., 42:47-50, 1974.

[13] Marshall Bowen and Joseph Coster. Methods of establishing the asymptotic behavior of the harmonic oscillator wave functions. Am. J. Phys., 48:307-308, 1980. 
[14] G. Solt and J.-P. Amiet. On the "series method" to solve Schrödinger's equation for the linear oscillator. Am. J. Phys., 53:180-181, 1985.

[15] Ira N. Levine. Molecular Spectroscopy. Wiley, New York, 1975.

[16] Gerhard Herzberg. Molecular Spectra and Molecular Structure. I: Spectra of Diatomic Molecules. Van Nostrand, Princeton, second edition, 1950.

[17] I. S. Gradshteyn and I. M. Ryzhik. Table of Integrals, Series, and Products. Academic Press, San Diego, fifth edition, 1994. 